\title{
IMPACT OF DIFFERENT UNLOADING ZONE LOCATIONS IN TRANSSHIPMENT TERMINALS UNDER VARIOUS FORKLIFT DISPATCHING RULES
}

\author{
Uwe Clausen \\ Jan Kaffka \\ Daniel Diekmann \\ Larissa Mest \\ TU Dortmund University \\ Institute of Transport Logistics \\ D 44227 Dortmund, GERMANY
}

\begin{abstract}
Operators of less-than-truckload terminals face the challenge of improving their efficiency to reduce handling costs and increase the performance of the terminal due to small profit margins. This paper uses material flow simulation to address the impact of different operational levers on a forklift-based internal transportation system. For a given I-shaped terminal two concepts of locating unloading zones are compared and evaluated concerning travel time of forklifts. In addition, different dispatching rules for the forklifts are implemented to reduce the empty travel time of the forklift and identify the potential for improvement based on a distance-optimized fleet control.
\end{abstract}

\section{INTRODUCTION}

Transshipment terminals of forwarding agencies operate as consolidation and deconsolidation nodes in LTL (less-than-truckload) transportation networks. During the afternoon hours, shipments in the region of the terminals are collected in the pre-carriage process with local traffic vehicles and brought to the terminals. In the ongoing outbound handling period the incoming local traffic shipments are sorted for specific long-distance line hauls. The consolidation of the shipments in the terminals increases the utilization of the line hauls and achieves a cost-efficient overnight transport. In the early morning hours the task of the transshipment terminal is the distribution of freight. The incoming line hauls are sorted for different local delivery routes. In the onward-carriage the shipments are transported to the consignee.

The transportation network normally enables the shipper to deliver national shipments in European countries in one day. However, the predefined periods for pre-carriage, line haul and onward-carriage result into traffic peaks inside the transshipment terminals around 5-7 pm during the outbound shift and 3-6 am during the inbound shift. At this period of time the transshipment terminals reach the performance limit which may lead to delayed processing or tardy departure. Shipments which cannot be processed in time for loading miss the follow-up action and are delivered one day late.

Besides the challenge of the outbound and inbound peaks, the operators of LTL transshipment terminals also have to deal with oddly shaped freight. Consequently, the capabilities of automated sorting and unloading technology are very limited and the handling process inside the LTL terminals is costintensive. Because there is hardly any potential in using different technical means, the optimization of strategies and operations is the most important task to increase the efficiency of the terminals. In addition, the daily handled amount of freight varies considerably and requires a flexible internal transport system. As a result, mostly forklifts are used to transport shipments inside the terminals. 
This paper focuses on the forklift-based internal sorting and transportation process to improve the system performance. The number of workers needed daily depends directly on the distances travelled to handle the shipments, thus travel times of the forklifts are used as key indicators to measure system performance. On the one hand a layout approach is evaluated by moving the unloading zone to different locations, which results into new source-sink-distances. On the other hand different dispatching strategies for forklift operations are implemented to study their impact on system performance.

\section{SYSTEM DESCRIPTION}

Generally, the area of a LTL terminal can be divided between the yard and the terminal building. The process in the yard starts with the registration of the arriving vehicles. Depending on the task (loading or unloading) and the utilization of the terminal, the vehicle is assigned to a door or is sent to a waiting place. The yard management controls the movement on the yard and dispatches waiting vehicles to doors.

Provided that the vehicle is loaded, it is assigned to the strip doors next to the unloading zone. The shipments are then moved to an unloading zone by freight carrier or terminal staff. Due to limited space in the unloading zone, the shipments remain in this area only for a short time. The time is used by the staff to match the shipment with the shipping documents. Additional work (e.g., labeling the shipments with barcodes) is completed in the unloading area.

The handling in the unloading area is completed and the shipment is transported to the loading buffer, where sorting of the palletized freight is done by forklifts. The loading buffers are predominantly used for one specific destination each. Yet based on the shortage of space, shared buffers for different routing destinations also appear, especially in local transport. Finally the shipments are transported from the loading buffers into the outgoing vehicles. Figure 1 illustrates the steps of the handling process in a LTL terminal. Concerning the complex interdependencies of the involved resources over time, an event-discrete simulation method is eminently suitable to analyze the stochastic system.

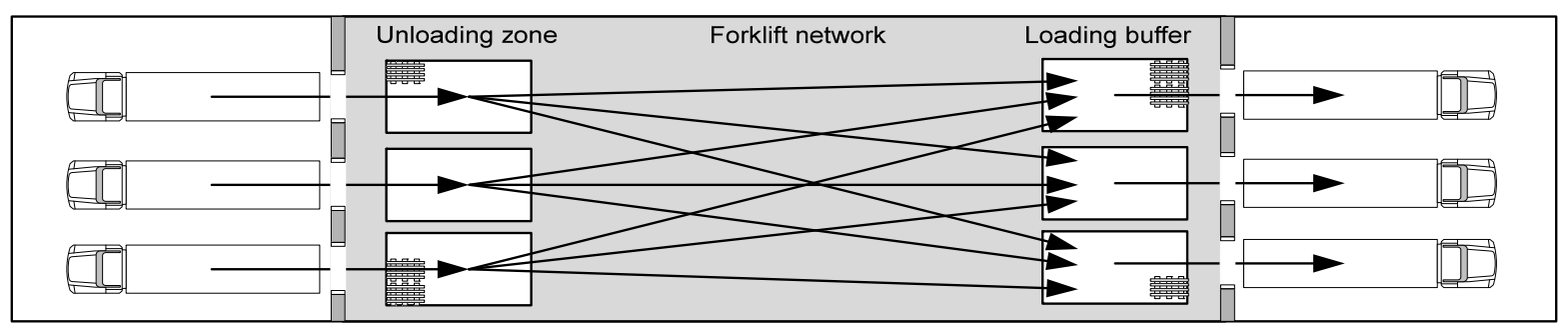

Figure 1: Handling processes in LTL terminals

\section{OPTIMIZING LTL TERMINALS}

Authors have proposed solutions to layout problems for freight terminals (Peck 1983; Tsui and Chang 1990, 1992), but in every case, freight flows from strip doors to destinations were assumed to be known and independent of internal travel paths and buffer spaces. Under such conditions, the supervisor's policy is equivalent to a "First Come First Served" policy (Gue 1995). If the supervisor assigns incoming trailers to strip doors, and manages forklifts between the functional areas based on the contents of the trailers waiting in the queue and the location of the doors, material flows depend on the layout. To optimize the assignment of trailers to load doors on the tactical level, Chmielewski (2007) developed a helpful doorassigner, using a column generation algorithm.

Gue and Bartholdi (2004) studied the impact of the shape of LTL terminals on labor cost. During their research they analyzed different terminals, commonly I, L, T-shaped. According to their results for small or mid-sized terminals with a maximum of 150 doors, I-shaped buildings perform best with respect 
of labor cost. With regard to the characteristics of national transportation systems, this is also the typical shape of German LTL terminals and is studied in this paper.

Aside from the shape of a terminal a key factor is the number of doors. To determine the number, a differentiated approach regarding strip and load doors is required. For the number of load doors the outgoing line hauls and local traffic tours define the number of doors, whereas the number of strip doors relates to parameters of daily throughput and average time of unloading a trailer. In this paper, we consider the shape and the number of strip and load doors as predefined by the real layout. Based on the given shape with a fixed number of doors, the variable component in the internal layout planning is the position of the unloading zones. To study the impact of the unloading zones positions, this paper analyzes two different layout concepts seen in figure 2 .

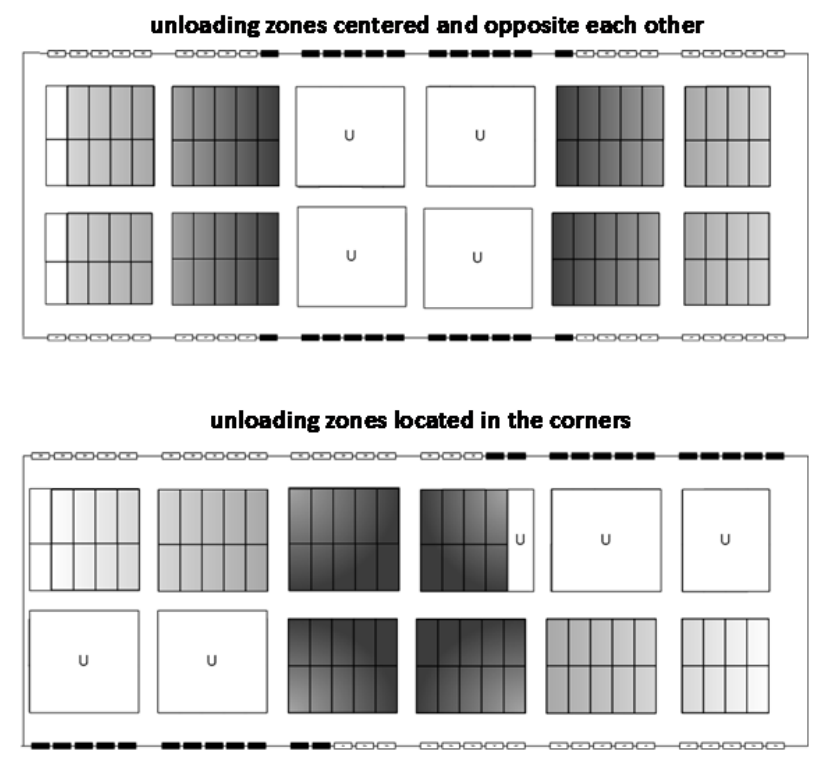

Figure 2: Position of unloading zones

In the first layout the unloading zones are placed in the center of the LTL terminal. Consistently strip doors are arranged next to the unloading zone opposite to each other. As a next step, the loading buffers for destinations with a high amount of shipments are positioned near the unloading zones in order to decrease the fork lift travel path. This arrangement of doors provides the shortest possible path for the forklift from the unloading zone to the loading buffers. However, the empty travel time in this variation is nearly the same as the full travel time. As a result, another possible arrangement of doors is tested in this paper. Based on the theory of building transportation cycles from one unloading zone to the other strip doors are placed at the opposite corners of the LTL terminal. The loading buffers for destinations with a large number of shipments are located in the center of the terminal, enabling forklifts to travel to the next door in a short period of time. Both layout concepts are tested under various dispatching strategies to determine the system impact.

\section{DISPATCHING RULES}

As described, a fleet of forklifts is used to execute the internal sorting and transportation process. Dispatching strategies have to be applied to control this system. For automated guided vehicles many strategies to determinate the routes and the actions of vehicle exist. According to Le-Anh and De Koster (2006), a distinction in the dispatching system is drawn between decentralized and centralized control systems. The main disadvantage of the decentralized system is low efficiency due to local optimization; in 
contrast, these control systems are very simple. Centralized control systems monitor all the vehicles and store the information in a database and are thus able to make more efficient assignments.

Egbelu and Tanchoco (1984) divide vehicle dispatching decisions into two categories. In the first place a dispatching decision has to be made when a forklift drops off a load and is ready for the next task. This assignment problem is initiated by the forklift and requires one or more transportation tasks. Provided that there is no transportation task available, the forklift remains idle until a new transportation task obtains the system and is assigned to the vehicle. If there is only one task waiting, a no choice assignment to this task is made. Conversely, a shipment, which needs to be transported to a sink, requires a dispatching decision to find a suitable forklift. This task-initiated assignment problem requires one or more free forklifts. In the event that all forklifts are busy, the transportation task is waiting for a free transporter. Again, if there is only one free transporter, a no choice assignment happens. Under the terms of the real case scenario, all forklifts are compatible with all palletized transportation tasks. Consequentially a system status, at which time more than one forklift and more than one transportation task are waiting for assignment, is not possible. Figure 3 depicts the task or forklift triggered ways of forklift dispatching.

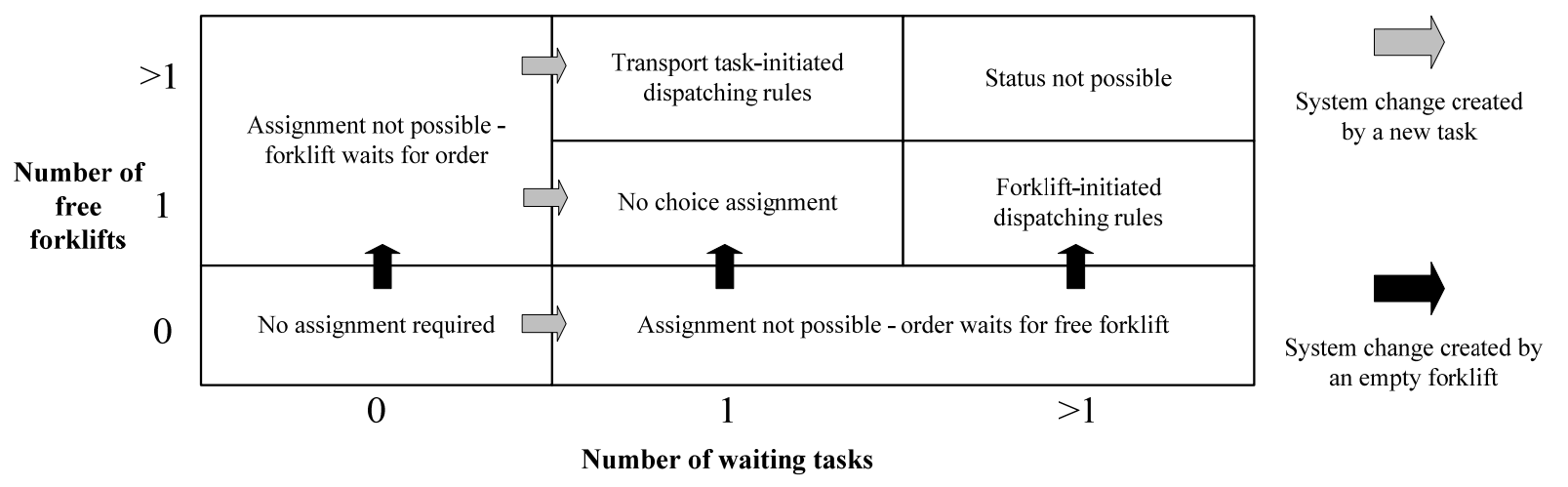

Figure 3: Event-based possibilities of forklift-task assignment

Another aspect of dispatching rules is the number of decision parameters. While single-attribute dispatching rules only have one criterion, multi-attribute rules use more than one parameter (Klein and Kim 1996). To measure the impact of improved strategies on the system of LTL terminals, single-attribute rules will be analyzed in the first step. Le-Anh and De Koster (2006) divides single-attribute dispatching rules into three main categories. Distance-based dispatching rules focus on travel distance or travel times. These rules reduce the empty travel times of the forklifts by assigning the nearest forklift or the nearest pickup point. Strategies that take filling level of the buffers into account are called workload-based dispatching rules. The aim of these rules is to reduce the number of overflowing queues. Occupied buffers lead to blockings in previous process steps. Furthermore, workload-based dispatching rules are also used for equal vehicle utilization. The last category of vehicle dispatching strategies takes the waiting time of the shipment into account. This includes the "First Come First Served" rule with its modifications. Further research with reassignment and look-ahead period strategies has shown additional potential to improve automated guided vehicle controls systems (e.g., Ichoua, Gendreau, and Potvin 2006). In practice, reassignment strategies cannot be applied to manual-guided forklift systems (Le-Anh and De Koster 2005) and detailed information about incoming vehicles and shipments for look-ahead strategies is not available.

In the simulation the following strategies for the forklifts in the transshipment terminals under various unloading areas are implemented.

Forklift-initiated rules: 
- LWT (Longest Waiting Time) - The transportation task with the longest waiting time is handled first. Due to access options of the buffer spaces, more than one move request is feasible, so no further modifications of the rule are necessary.

- STDPP (Shortest Travel Distance Pickup Point) - This rule assigns the task with the shortest travel distance. Once the forklift is ready for the next order, the task with the closest pickup point is assigned first.

- MaxQS (Maximum Queue Size) - A transportation task in the buffer with the largest number of task waiting is selected first.

Task-initiated rules:

- FTF (First Transporter First) Under this basic rule the first free transporter is assigned to the transportation task.

- NV (Nearest Vehicle) - This rule searches for the nearest vehicle of all free transporters.

- LUV (Least Utilized Vehicle) - The transportation task is assigned to the forklift with the lowest utilization.

\section{MODELING AND EXPERIMENTAL PLANNING}

To analyze the interaction and effects of strategic planning (e.g., layout decisions) on operational procedures, the Chair of Transportation Systems and Logistics, TU Dortmund University, has developed a transportation logistics simulation environment. Based on the simulation software Enterprise Dynamics, the tool ED Transport permits the illustration and simulation of material and information flows in logistic nodes (e.g., transshipment terminals and distribution centers) of road transport (Neumann and Deymann 2008). ED Transport provides the possibility to model a LTL terminal on a microscopical layer. All handling and controlling processes of the terminal can be modeled and parameterized (arrival of a truck at a gate, unloading, transporting, buffering, loading, departure of a truck).

The terminal used for testing of dispatching and layout strategies has the following scope:

- Ground space of $6300 \mathrm{~m}^{2}$

- 24 unloading doors and 36 loading doors

- 4300 Handling units per day for inbound and outbound

- 23 national and 33 regional destinations

The basic layout of the terminal with its doors, (un)loading and buffer areas is drawn to scale and is modeled with ED Transport, as seen in Figure 4.

The differences between the two layout concepts are the functions of doors and the resulting doordestination assignments. The modeling of the surrounding yard system contains a module to control and track the movements which occur in the yard, including door or waiting place assignment, called Yard Management. Arriving vehicles are created with a generator based on the real data. The vehicles are loaded with shipments, which contain labeled destination information. After arriving at the gate, the vehicles are sent either to a waiting area or directly to a door. This depends on the availability of a suitable door.

Vehicles for unloading can be docked at every free door for unloading. The Yard Management of ED Transport allocates the vehicles to the door based on an equal distribution. Loading vehicles can only be docked at their preferred door or at specified alternative doors. Based on this door-destinationassignment, the Yard Management always tries at first to allocate the vehicle to its preferred door. If this door is not available, alternative doors are checked for availability. If none of the possible doors are free for (un-)loading, it is allocated to the waiting area. When a vehicle is fully loaded and undocks from the door, the Yard Management checks at first the waiting area for vehicles waiting for this specific door. Only if no vehicle can be found is the door released for new arrivals. 


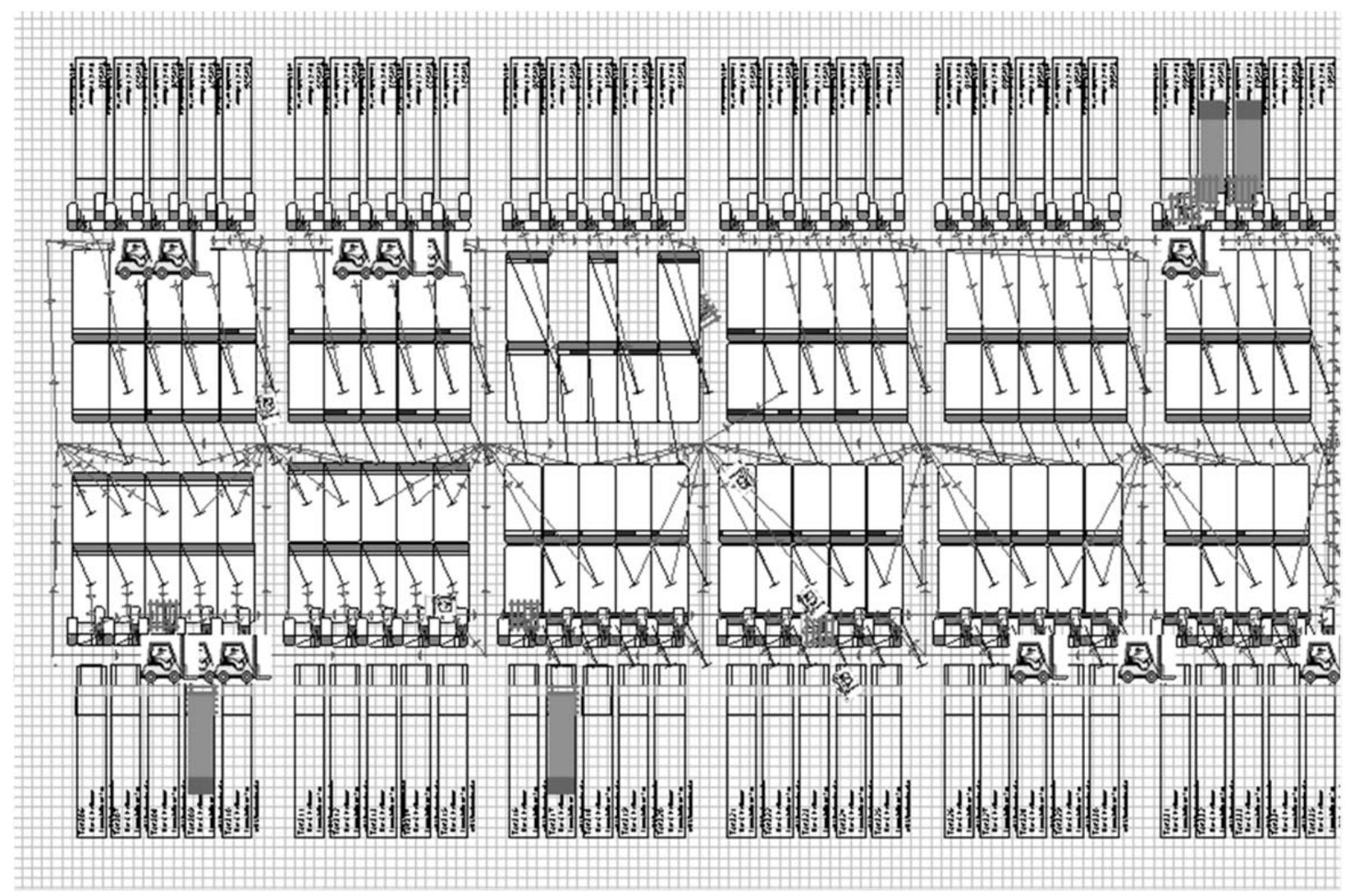

Figure 4: Simulation model

For internal transportation forklifts use a bidirectional network based on the real internal transport path. Every forklift has a capacity of one shipment. Shipments are dispatched through this network of nodes and edges, calculating the shortest path with Dijkstra Algorithm. In this network all dispatching strategies described above are implemented for forklifts. The time for handling processes, except the dispatching of shipments, is modeled with distribution functions based on time measurements. Due to the specific characteristics of the terminal, not all dispatching strategies described above are reasonable for the scope of this paper. The focus of this study is to reduce costs by lowering transport time and distance. Consequently, the forklift-initiated rule MaxQS, and the task-initiated rule LUV, are not examined in this paper. Both strategies do not aim at reducing transportation distances.

Thus, in this paper, two forklift-initiated rules (LWT and STDPP) and two task-initiated rules (FTF and NV) are tested. All possible combinations are:

- FTF-LWT

- FTF-STDPP

- NV-LWT

- NV-STDPP

For the two different layout concepts, all four combination are considered, which results in eight different scenarios.

Forwarding agencies usually do not work with a forklift fleet control; as a result the first-come-firstserved combination FTF-LWT is considered as the basic scenario, because other dispatching strategies allocate tasks to the forklifts distance-optimized. Accordingly, this requires a forklift fleet control which regulates positions of the forklifts and shipments and allocates tasks to forklifts in an optimized way. 
The simulation time is one working week (five days) with a start-up period of one day. To avoid statistically questionable values every simulation scenario is repeated 20 times. All results are average values of these multi simulation runs.

\section{RESULTS}

Forklifts always work in a process cycle, that is, as a task appears, the forklift travels empty to the source, picks up the shipment and travels fully loaded to the sink based on its destination and drops off the shipment. After that, the forklift waits for a new task. Based on the scope of the paper, the travel times are the relevant key figures to analyze. The full travel time in one layout concept cannot be optimized, because the destination of a shipment is always the same and the door-destination-assignment is not changed. So full travel times can only be influenced when the layout is changed. Thus, the full travel time is an indicator for validating the comparability of different scenarios within the different layout concepts opposite sies and corners, shown in Figure 2.

As illustrated in Figure 5, the average full travel time in the different scenarios is constant. Despite the different docking of trucks at the strip doors, and the different location of shipments in the unloading zone, the different scenarios are comparable.

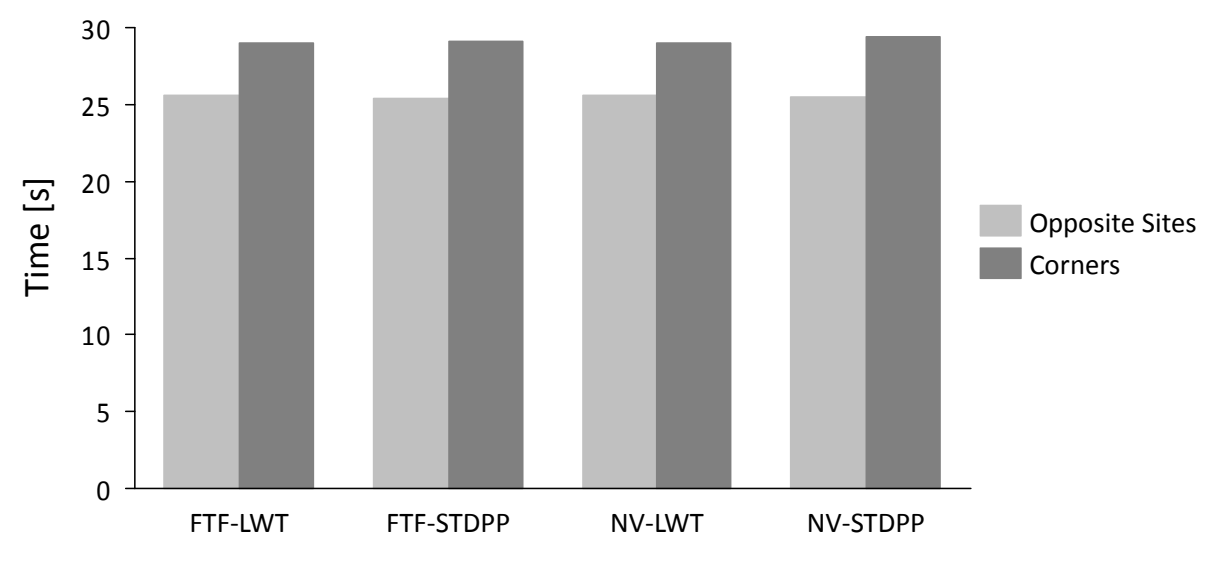

Figure 5: Average full travel time per shipment

Only observing the full travel time, the position of the unloading zone in the center and divided into two zones located on opposite sites is 12 percent faster per shipment than the corner concept. By applying the corner concept, the full travel path is lengthened. In an effort to reduce the empty travel time an examination of the empty travel time is necessary.

Figure 6 shows the average empty travel time with its percentage changes in relation to the FTF-LWT strategy.

The change of the forklift initiated rules from LWT to STDPP provides an improvement in empty travel time of 10 percent and 13 percent per shipment depending on the layout concept. The change of task-initiated rules from FTF to NV improves system performance from 8 percent to 11 percent, while the change of both rules improves performance from 17 percent to 26 percent. The comparison of both layout concepts shows that the corner concept is slower than the opposite site variation in each scenario. This is due to fact that the number of possible cycles with a short travel time to the next corner is not as high as expected. Often the situation occurs that the next transportation task is located in the original loading zone, which besides creating a longer full travel way, results in a longer empty travel way. Moreover, the location of the loading buffers for destinations with a large number of shipments in the middle of the Ishaped terminal produces for a high amount of shipments the same empty travel times as in the opposite sites layout. 


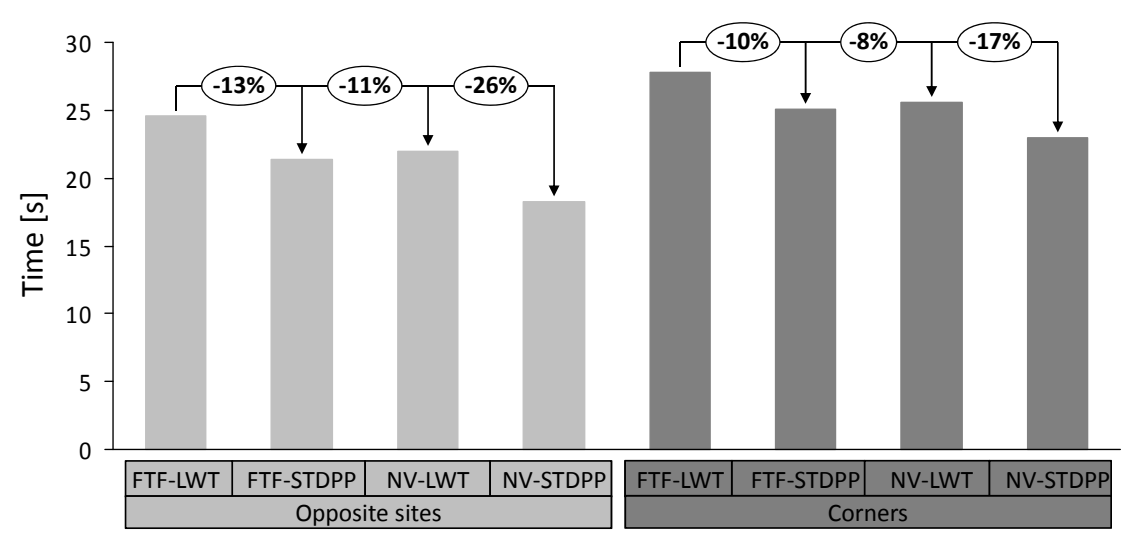

Figure 6: Average empty travel time per shipment

To examine more closely the improvement resulting from changing the dispatching strategies, Figure 7 shows a box plot of the basic and the NV-STDPP scenarios regarding the opposite sites layout.

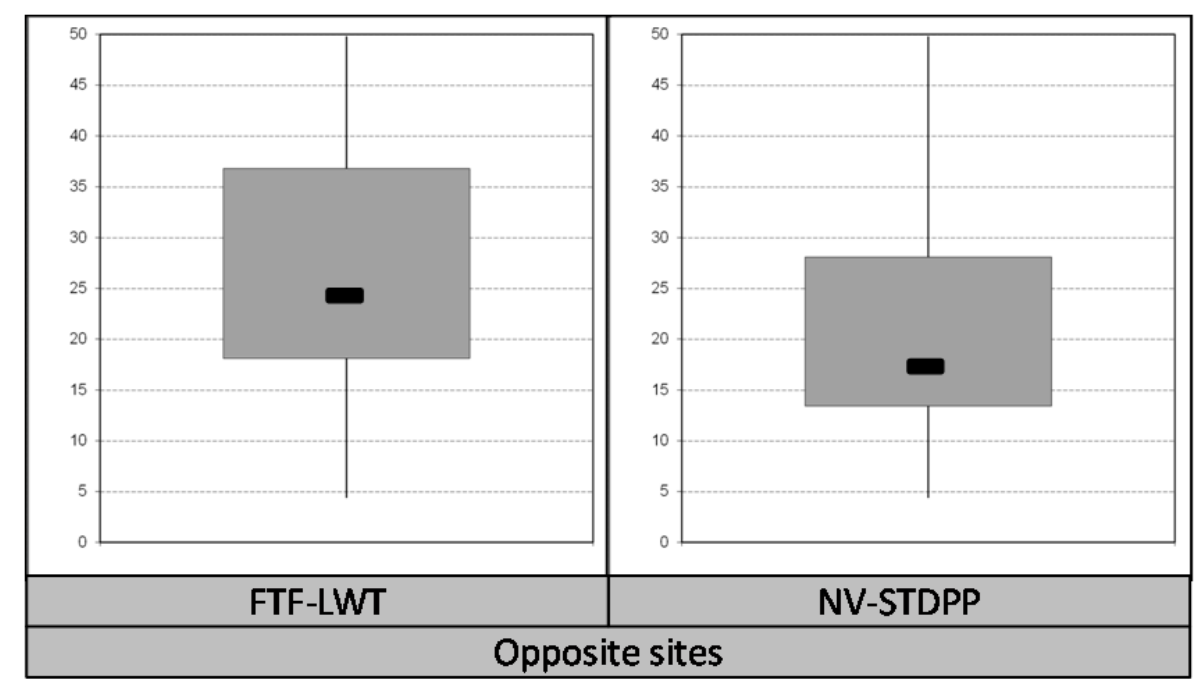

Figure 7: Box Plot comparison

In addition to decreasing the average empty travel time by 26 percent, the strategy change also decreases the upper and lower quartile of the value allocation. The spread of the values is thus lowered and the transportation process of the LTL terminal is more stable. Comparing these two scenarios the NVSTDPP strategies save on average eight hours of travel time. As a result, one forklift can be saved, leading to cost reduction or deployed for other value-added work.

The transportation processes not only affect the variation of travel times; it also has an influence on availability of doors and departing times of vehicles. The unloading zone is limited in its capacity by its size. When the zone is not at full capacity, shipments can constantly be unloaded from a vehicle into the zone and the time a shipment remains in the zone has no influence on this process. This changes when the zone reaches its limit. The faster the shipments leave the unloading zone, the faster a vehicle can be unloaded and leave the door. Another factor is the availability of shipments for loading. Shipments which remain for a shorter period in the unloading zone, are available earlier for loading and vehicles can depart earlier. Figure 8 shows a comparison of the average waiting time of a shipment in an unloading zone. 
By changing the forklift-initiated rules from LWT to STDPP, the waiting time can be reduced by 88 percent in the opposite site layout concept and 60 percent in the corners layout. This key figure also shows a significant advantage of the opposite site layout concept of 85 percent compared to the corner concept.

\section{CONCLUSION}

This paper demonstrates that a layout concept for I-shaped LTL terminals with unloading zones in the center of the terminal is significantly better than a concept with unloading zones in opposite corners of the terminal. Furthermore, a travel path optimizing forklift fleet control reduces the empty travel time of the forklifts compared to the currently used FTF-LWT concept by up to 26 percent. However, implementing such a control system requires process changes in the terminal and additional investments. The control system always needs to know the positions of shipments and forklifts. To achieve this it is necessary that every shipment is scanned after unloading in unloading zone. The forklift driver must at least scan a sign with a barcode at the unloading zone to report his position to the control system when dropping off a shipment. Moreover, the system can be upgraded to an automatic location detection.

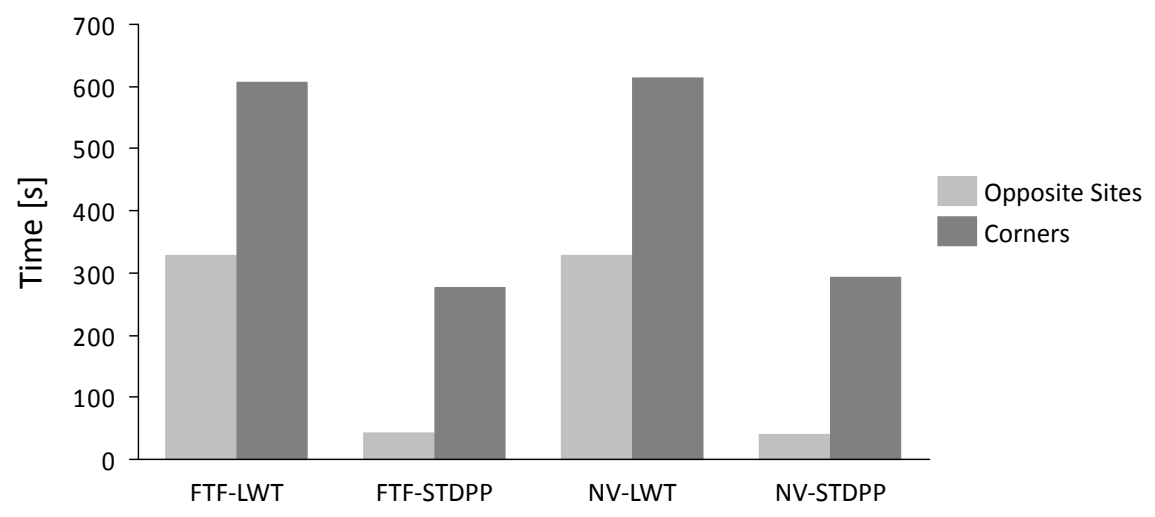

Figure 8: Average time of a shipment in unloading zones

A topic of future work will be the extension of the single to multi attribute strategies as fleet control has a large impact on terminal performance. With regard to LTL terminals rush orders in particular which are caused by tardy vehicles, have to be dispatched first using a higher priority number.

\section{REFERENCES}

Chmielewski, A. 2007. "Entwicklung optimaler Torbelegungspläne in Stückgutspeditionsanlagen.” Ph.D. thesis, TU Dortmund University.

Egbelu, P. J., and J. M. A. Tanchoco. 1984. "Characterization of Automated Guided Vehicle Dispatching Rules.” Int. J. Prod. Res. 22:359-374.

Gue, K. R. 1995. "Freight Terminal Layout and Operations." Ph.D. thesis, Georgia Institute of Technology.

Gue, K. R., and J. Bartholdi. 2004. "The Best Shape for a Crossdock." Transportation Research 38(2):235-244.

Ichoua, S., M. Gendreau, and J.-Y. Potvin. 2006. "Exploiting Knowledge About Future Demands for Real-Time Vehicle Dispatching." Transportation Science 40(2):211-225.

Klein, C. M., and J. Kim. 1996. “AGV Dispatching.” Int. J. Prod. Res. 34:95-110.

Le-Anh, T. and M. B. M. De Koster. 2005. "On-line Dispatching Rules For Vehicle-Based Internal Transport Systems." International Journal of Production Research 43(8):1711-1728. 
Le-Anh, T., and M. B. M. De Koster. 2006. "A Review of Design and Control of Automated Guided Vehicle Systems." European Journal of Operational Research 171:1-23.

Neumann, L. and S. Deymann. 2008. "Transsim-Node - A Simulation Tool for Logistics Nodes.” In Proceeding of the2008 Industrial Simulation Conference, edited by J. Colloc, C. Petit and C. Dussart, 283-287. The European Simulation Society.

Peck, K. E. 1983. "Operational Analysis of Freight Terminals Handling Less Than Container Load Shipments." Ph.D. thesis, University of Illinois at Urbana-Champaign.

Tsui, L.-Y., and C.-H. Chang. 1990. "A Microcomputer Based Decision Support Tool for Assigning Dock Doors in Freight Yards." Comput. Indust. Eng. 19:309-312.

Tsui, L.-Y., and C.-H. Chang. 1992. "Optimal Solution to a Dock Door Assignment Problem." Comput. Indust. Eng. 23:283-286.

\section{AUTHOR BIOGRAPHIES}

UWE CLAUSEN is the managing director of the Institute of Transport Logistics at the TU Dortmund University and director of the Fraunhofer-Institute for Material Flow and Logistics (IML). Prof. Dr.-Ing. Clausen studied computer sciences at the University of Karlsruhe (TH) and finished his doctoral thesis on transportation network optimization at TU Dortmund University in 1995. Afterwards, he worked at the "Deutsche Post AG" and joined Amazon.de in 1999. As operations director he was responsible for setting up the new Amazon Logistics Center in Bad Hersfeld. In 2001 Uwe Clausen accepted the chair "Transport Systems and Logistics" at TU Dortmund University, which became the Institute of Transport Logistics in 2011. He is amongst others chairman of EffizienzCluster LogistikRuhr and a member of ECTRI European Conference of Transport Research Institutes and of the Scientific Advisory Board of German Logistics Association (BVL). His e-mail address is clausen@itl.tu-dortmund.de.

JAN KAFFKA was born in Münster, Germany, and attended TU Dortmund University, where he studied logistics and obtained his degree in 2008. He works at the Institute of Transport Logistics at TU Dortmund University as a scientific assistant and as a full-time $\mathrm{PhD}$ student, doing research in the fields of intermodal freight transport and simulation in traffic and transport logistics. His e-mail address is kaffka@itl.tu-dortmund.de.

DANIEL DIEKMANN was born in Melle, Germany, and attended TU Dortmund University, where he studied logistics and obtained his degree in 2010. He works at the Institute of Transport Logistics at TU Dortmund University as a scientific assistant and as a full-time PhD student, doing research in the fields of sorting systems and simulation in traffic and transport logistics. His e-mail address is diekmann@itl.tudortmund.de.

LARISSA MEST was born in Moers, Germany, and attended TU Dortmund University, where she studied logistics and obtained her degree in 2004. She works at the Institute of Transport Logistics at TU Dortmund University as a scientific assistant and as a full-time $\mathrm{PhD}$ student, doing research in the fields of commercial traffic and simulation in traffic and transport logistics. Her e-mail address is mest@itl.tudortmund.de. 